\title{
REDUCTION OF QUAD-EQUATIONS CONSISTENT AROUND A CUBOCTAHEDRON I: ADDITIVE CASE
}

\author{
NALINI JOSHI AND NOBUTAKA NAKAZONO
}

(Communicated by Mourad Ismail)

\begin{abstract}
In this paper, we consider a reduction of a new system of partial difference equations, which was obtained in our previous paper [Classification of quad-equations on a cuboctahedron, arXiv:1906:06650,2019] and shown to be consistent around a cuboctahedron. We show that this system reduces to $A_{2}^{(1) *}$-type discrete Painlevé equations by considering a periodic reduction of a three-dimensional lattice constructed from overlapping cuboctahedra.
\end{abstract}

\section{INTRODUCTION}

In this paper, we consider a system of partial difference equations ( $\mathrm{P} \Delta \mathrm{Es})$ governing a function $u=u(\boldsymbol{l})$ taking values on the vertices of a face-centered cubic lattice $\Omega$, given by

$$
\Omega=\left\{\boldsymbol{l}=\sum_{i=1}^{3} l_{i} \boldsymbol{\epsilon}_{i} \mid l_{i} \in \mathbb{Z}, l_{1}+l_{2}+l_{3} \in 2 \mathbb{Z}\right\},
$$

where $\left\{\boldsymbol{\epsilon}_{1}, \boldsymbol{\epsilon}_{2}, \boldsymbol{\epsilon}_{3}\right\}$ is a standard basis of $\mathbb{R}^{3}$. The system consists of 6 equations:

$$
\frac{u_{\overline{i k}}}{u_{\underline{i} \bar{k}}}=\frac{\left(\alpha_{i j}+\gamma_{i}\right) u_{\overline{j k}}-\left(\alpha_{i j}+\gamma_{j}-\gamma_{k}\right) u_{\underline{j} \bar{k}}}{\left(\alpha_{i j}-\gamma_{j}+\gamma_{k}\right) u_{\overline{j k}}-\left(\alpha_{i j}-\gamma_{i}\right) u_{\underline{j} \bar{k}}}, \frac{u_{j k}}{u_{\overline{j k}}}=\frac{\left(\alpha_{i j}+\gamma_{i}\right) u_{\underline{i k}}-\left(\alpha_{i j}-\gamma_{j}+\gamma_{k}\right) u_{\overline{i k}}}{\left(\alpha_{i j}+\gamma_{j}-\gamma_{k}\right) u_{\underline{i k}}-\left(\alpha_{i j}-\gamma_{i}\right) u_{\overline{i k}}},
$$

where $(i, j, k)=(1,2,3),(2,3,1),(3,1,2)$, and the bars $\bar{i}$ and $j$ denote $\boldsymbol{l} \rightarrow \boldsymbol{l}+\boldsymbol{\epsilon}_{i}$ and $\boldsymbol{l} \rightarrow \boldsymbol{l}-\boldsymbol{\epsilon}_{j}$ respectively and the coefficients are given by

$$
\begin{array}{lll}
\alpha_{i j}=\alpha_{i}\left(l_{i}\right)-\alpha_{j}\left(l_{j}\right), & \alpha_{i}(k)=\alpha_{i}(0)+k, & i, j \in\{1,2,3\}, k \in \mathbb{Z}, \\
\gamma_{1}=-c+(-1)^{l_{1}+l_{2}} \delta_{1}, & \gamma_{2}=-c+(-1)^{l_{2}+l_{3}} \delta_{2}, & \gamma_{3}=-c+(-1)^{l_{1}+l_{3}} \delta_{3},
\end{array}
$$

with $\alpha_{i}(0), i=1,2,3, c$, and $\delta_{j}, j=1,2,3$, being complex parameters. Figure 1.1 shows a unit cell in $\Omega$.

Received by the editors June 28, 2020.

2020 Mathematics Subject Classification. Primary 33E30, 34M55, 37K10, 39A14, 39A23, 39A45.

Key words and phrases. Consistency around a cuboctahedron, consistency around an octahedron, quad-equation, consistency around a cube, ABS equation, discrete Painlevé equation.

This research was supported by an Australian Laureate Fellowship \# FL120100094 and grant \# DP160101728 from the Australian Research Council and JSPS KAKENHI Grant Numbers JP19K14559 and JP17J00092. 


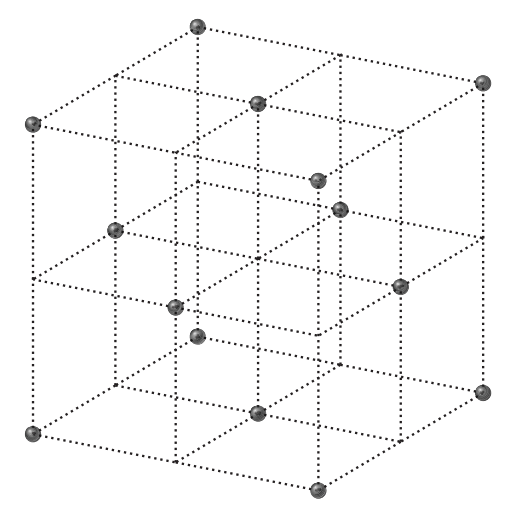

Figure 1.1. A unit cell of the $\Omega$ lattice

Our study is motivated by two considerations. Firstly, the system (1.2) satisfies the consistency around a cuboctahedron (CACO) property [10, which is a generalization of the famous consistency around a cube (CAC) property [17]. (See Appendix $\mathrm{A}$ for a summary of the details of the CACO property and $\$ 1.2$ for those of the CAC property.) Secondly, we are motivated by finding relations between partial difference equations and ordinary difference equations known as the discrete Painlevé equations.

In this paper, we show that the system (1.2) reduces to discrete Painlevé equations with initial value space characterised as $A_{2}^{(1) *}$ in the sense of Sakai [22]. The latter equations have two forms in the literature given respectively by Tsuda [23] and Ramani et al. 21] and are explicitly given by:

$$
\begin{aligned}
& \left\{\begin{array}{l}
(\underline{Y}+X)(X+Y)=\frac{\left(\left(X+c_{3}\right)^{2}-c_{1}\right)\left(\left(X-c_{3}\right)^{2}-c_{2}\right)}{(X+t)^{2}-c_{4}} \\
(\bar{X}+Y)(X+Y)=\frac{\left(\left(Y-c_{3}\right)^{2}-c_{1}\right)\left(\left(Y+c_{3}\right)^{2}-c_{2}\right)}{\left(Y+t+\frac{1}{2}\right)^{2}-c_{5}}
\end{array}\right. \\
& (\bar{X}+X)(\underline{X}+X)=\frac{\left(X^{2}-c_{1}\right)\left(X^{2}-c_{2}\right)}{(X+t)^{2}-c_{3}} .
\end{aligned}
$$

Here, $t \in \mathbb{C}$ is an independent variable, $c_{i}, i=1 \ldots, 5$, are complex parameters and $X, Y$ are dependent variables:

$$
X=X(t), \quad Y=Y(t), \quad \bar{X}=X(t+1), \quad \underline{X}=X(t-1), \quad \underline{Y}=Y(t-1) .
$$

We note that discrete Painlevé equations admit special solutions when parameters take special values. For example, Equation (1.4a) has the special solution given by the generalized hypergeometric series ${ }_{3} F_{2}$ when $4 c_{3}+2 \sqrt{c_{4}}+2 \sqrt{c_{5}}=1$ [11.

Our main result is Theorem 1.1. To state the theorem, we first explain how to take the reduction on the lattice $\Omega$. To be explicit, consider a vertex $\boldsymbol{l} \in \Omega$, given by $l_{1} \epsilon_{1}+l_{2} \epsilon_{2}+l_{3} \epsilon_{3}$. Define the plane $H_{k} \subset \Omega$ given by $l_{3}=k$. We project the vertices of $H_{1}$ to the adjacent horizontal plane $H_{0}$ by taking $\left(l_{1}, l_{2}, 1\right) \mapsto\left(l_{1}-1, l_{2}-1,0\right)$. The union of the projection with the lattice points on $H_{0}$ forms $\mathbb{Z}^{2}$. We can define such a projection from every plane $H_{k}$ to $H_{0}$ by the following:

$$
\left(l_{1}, l_{2}, k\right) \mapsto\left(l_{1}-k, l_{2}-k, 0\right) .
$$


We call the result of this operation a $(1,1,1)$-periodic reduction.

Theorem 1.1. The $A_{2}^{(1) *}$-type discrete Painlevé equations (1.4) can be obtained from the system of $P \Delta E s$ (1.2) via the $(1,1,1)$-periodic reduction.

1.1. Notation and definitions. Throughout the paper, we use terminology to describe polynomials and quad-equations that is common in the literature. Readers who are unfamiliar with this notation may wish to consult $[1,8,10$. We use $Q=$ $Q(x, y, z, w)$ to denote a multivariable polynomial over $\mathbb{C}$. Under certain conditions, i.e., $Q$ be affine linear and irreducible, we will refer to the equation $Q=0$ as a quad-equation or sometimes, for succinctness, refer to the polynomial $Q$ as a quadequation. We remind the reader that the condition of irreducibility implies that $Q(x, y, z, w)=0$ can be solved for each argument, and that the solution is a rational function of the other three arguments.

1.2. Background. Integrable systems are widely applicable models of science, occurring in fluid dynamics, particle physics and optics. The prototypical example is the famous Korteweg-de Vries (KdV) equation whose solitary wave-like solutions interact with elastically like particles, leading to the invention of the term soliton. It is then natural to ask what discrete versions of such equations are also integrable. This question turns out to be related to consistency conditions for polynomials associated to faces of cubes as we explain below.

Integrable discrete systems were discovered [15, 16, 18, 20] from mappings that turn out to be consistent on multi-dimensional cubes. (We note that there are additional systems that do not fall into this class; see e.g., [8, Chapter 3].) These are quad-equations in the sense in \$1.1. In [1 4, Adler-Bobenko-Suris et al. classified quad-equations satisfying the consistency around a cube (CAC) property, which lead to integrable $\mathrm{P} \Delta \mathrm{Es}$. We refer to such $\mathrm{P} \Delta \mathrm{Es}$ as $\mathrm{ABS}$ equations. It turns out that ABS equations contain many well known integrable P $\Delta$ Es [9, 14, 16].

Reductions of integrable PDEs lead to Painlevé equations, which first arose in the search for new transcendental functions in the early 1900's [5, 6, 19. Again a natural question is to ask whether discrete versions exist with analogous properties. This question led to the discovery of second-order difference equations called the discrete Painlevé equations [7, 13, 20].

It is now well-known that discrete Painlevé equations have initial value spaces with geometric structures that can be identified with root systems and affine Weyl groups [22]. Sakai showed that there are 22 types of initial value spaces as shown in Table 1.1 .

TABLE 1.1. Types of spaces of initial values

\begin{tabular}{|l|l|}
\hline Discrete type & Type of space of initial values \\
\hline Elliptic & $A_{0}^{(1)}$ \\
\hline Multiplicative & $A_{0}^{(1) *}, A_{1}^{(1)}, A_{2}^{(1)}, A_{3}^{(1)}, \ldots, A_{8}^{(1)}, A_{7}^{(1)^{\prime}}$ \\
\hline Additive & $A_{0}^{(1) * *}, A_{1}^{(1) *}, A_{2}^{(1) *}, D_{4}^{(1)}, \ldots, D_{8}^{(1)}, E_{6}^{(1)}, E_{7}^{(1)}, E_{8}^{(1)}$ \\
\hline
\end{tabular}

1.3. Outline of the paper. This paper is organized as follows. In $₫ 2$ we show the extended affine Weyl group of type $E_{6}^{(1)}$ and its subgroup which forms that of 
type $A_{2}^{(1)}$. Moreover, from those birational actions we obtain the discrete Painlevé equations (1.4) and the P $\Delta$ Es (2.16), which are periodically reduced equations of the system (1.2). In $\$ 3$, using the results in $₫ 2$ we give the proof of Theorem 1.1 . Finally, we give some concluding remarks in 4 .

\section{Derivation of the Discrete integrable Systems From An EXtended AFFINE WEYL GROUP OF TYPE $E_{6}^{(1)}$}

In this section, we derive the partial/ordinary discrete integrable systems from the birational actions of an extended affine Weyl group of type $E_{6}^{(1)}$, denoted by $\widetilde{W}\left(E_{6}^{(1)}\right)$. Note that details of $\widetilde{W}\left(E_{6}^{(1)}\right)$ are given in Appendix B.

2.1. Extended affine Weyl group of type $A_{2}^{(1)}$. Let $a_{i}, i=0, \ldots, 6$, be parameters satisfying the condition

$$
a_{1}+2 a_{2}+3 a_{3}+2 a_{4}+a_{5}+2 a_{6}+a_{0}=1,
$$

and $\tau_{j}^{(i)}, i=1,2,3, j=0,1,2,3$, be variables. Moreover, we define the transformations $s_{i}, i=0, \ldots, 6, \iota_{j}, j=1,2,3$, by isomorphisms from the field of rational functions $K\left(\left\{\tau_{j}^{(i)}\right\}\right)$, where $K=\mathbb{C}\left(\left\{a_{i}\right\}\right)$, to itself. These transformations collectively form the extended affine Weyl group of type $E_{6}^{(1)}$, denoted by $\widetilde{W}\left(E_{6}^{(1)}\right)$ :

$$
\widetilde{W}\left(E_{6}^{(1)}\right)=\left\langle s_{0}, \ldots, s_{6}\right\rangle \rtimes\left\langle\iota_{1}, \iota_{2}, \iota_{3}\right\rangle .
$$

See Appendix B for more details.

Let us define the transformations $w_{i}, i=0,1,2$, and $\pi$ by

$$
w_{0}=s_{2} s_{1} s_{3} s_{2}, \quad w_{1}=s_{4} s_{5} s_{3} s_{4}, \quad w_{2}=s_{6} s_{0} s_{3} s_{6}, \quad \pi=\iota_{3} \iota_{1} .
$$

They collectively form the extended affine Weyl group of type $A_{2}^{(1)}$ :

$$
\widetilde{W}\left(A_{2}^{(1)}\right)=\left\langle w_{0}, w_{1}, w_{2}\right\rangle \rtimes\langle\pi\rangle .
$$

Indeed, the following fundamental relations hold:

$$
\left(w_{i} w_{j}\right)^{a_{i j}}=1, \quad i, j \in\{0,1,2\}, \quad \pi^{3}=1, \quad \pi w_{\{0,1,2\}}=w_{\{1,2,0\}} \pi,
$$

where

$$
\left(a_{i j}\right)_{i, j=0}^{2}=\left(\begin{array}{lll}
2 & 3 & 3 \\
3 & 2 & 3 \\
3 & 3 & 2
\end{array}\right)
$$

Introduce the parameters and variables that go well with $\widetilde{W}\left(A_{2}^{(1)}\right)$ as follows. Let

$$
b_{0}=a_{1}+2 a_{2}+a_{3}, \quad b_{1}=a_{3}+2 a_{4}+a_{5}, \quad b_{2}=a_{3}+2 a_{6}+a_{0},
$$

$$
c=\frac{a_{0}+a_{1}+a_{3}+a_{5}}{2}, \quad d_{12}=\frac{a_{0}+a_{1}-a_{3}-a_{5}}{2}, \quad d_{23}=\frac{a_{0}-a_{1}+a_{3}-a_{5}}{2},
$$

$$
d_{13}=\frac{a_{0}-a_{1}-a_{3}+a_{5}}{2},
$$


where $b_{0}+b_{1}+b_{2}=1$, and

$$
y_{1}=\frac{\tau_{1}^{(1)}}{\tau_{0}^{(1)}}, \quad y_{2}=\frac{\tau_{3}^{(3)}}{\tau_{2}^{(3)}}, \quad y_{3}=\frac{\tau_{1}^{(2)}}{\tau_{0}^{(2)}}, \quad y_{4}=\frac{\tau_{3}^{(2)}}{\tau_{2}^{(2)}}, \quad y_{5}=\frac{\tau_{3}^{(1)}}{\tau_{2}^{(1)}}, \quad y_{6}=\frac{\tau_{1}^{(3)}}{\tau_{0}^{(3)}}
$$

Then, the actions of $\widetilde{W}\left(A_{2}^{(1)}\right)$ on the parameters $b_{0}, b_{1}, b_{2}, c, d_{12}, d_{23}, d_{13}$ are given by

$$
\begin{aligned}
& w_{i}\left(b_{j}\right)=\left\{\begin{array}{ll}
-b_{i} & \text { if } i=j, \\
b_{j}+b_{i} & \text { if } i \neq j,
\end{array} \quad w_{0}:\left(d_{12}, d_{23}\right) \mapsto\left(d_{23}, d_{12}\right),\right. \\
& w_{1}:\left(d_{23}, d_{13}\right) \mapsto\left(d_{13}, d_{23}\right), \\
& \pi:\left(b_{0}, b_{1}, b_{2}, d_{12}, d_{23}, d_{13}\right) \mapsto\left(b_{1}, b_{2}, b_{0},-d_{23},-d_{13}\right) \mapsto\left(-d_{13},-d_{12}\right),
\end{aligned}
$$

where $i, j \in \mathbb{Z} /(3 \mathbb{Z})$, while those on the $y$-variables $y_{i}, i=1, \ldots, 6$, are given by

$$
\begin{gathered}
w_{0}:\left(\begin{array}{l}
y_{1}, y_{3} \\
y_{5}, y_{6}
\end{array}\right) \mapsto\left(\begin{array}{c}
y_{5}, \frac{\left(b_{0}-c+d_{13}\right) y_{3}-\left(b_{0}-d_{12}+d_{23}\right) y_{1}}{\left(b_{0}+d_{12}-d_{23}\right) y_{3}-\left(b_{0}+c-d_{13}\right) y_{1}} y_{5} \\
y_{1}, \frac{\left(b_{0}-c+d_{13}\right) y_{6}-\left(b_{0}-d_{12}+d_{23}\right) y_{1}}{\left(b_{0}+d_{12}-d_{23}\right) y_{6}-\left(b_{0}+c-d_{13}\right) y_{1}} y_{5}
\end{array}\right), \\
w_{1}:\left(\begin{array}{l}
y_{1}, y_{3} \\
y_{4}, y_{6}
\end{array}\right) \mapsto\left(\begin{array}{l}
\frac{\left(b_{1}-c+d_{12}\right) y_{1}-\left(b_{1}-d_{13}+d_{23}\right) y_{3}}{\left(b_{1}+d_{13}-d_{23}\right) y_{1}-\left(b_{1}+c-d_{12}\right) y_{3}} y_{4}, y_{4} \\
y_{3}, \frac{\left(b_{1}-c+d_{12}\right) y_{6}-\left(b_{1}-d_{13}+d_{23}\right) y_{3}}{\left(b_{1}+d_{13}-d_{23}\right) y_{6}-\left(b_{1}+c-d_{12}\right) y_{3}} y_{4}
\end{array}\right), \\
w_{2}:\left(\begin{array}{l}
y_{1}, y_{2} \\
y_{3}, y_{6}
\end{array}\right) \mapsto\left(\begin{array}{l}
\frac{\left(b_{2}-c-d_{23}\right) y_{1}-\left(b_{2}-d_{12}-d_{13}\right) y_{6}}{\left(b_{2}+d_{12}+d_{13}\right) y_{1}-\left(b_{2}+c+d_{23}\right) y_{6}} y_{2}, y_{6} \\
\frac{\left(b_{2}-d_{12}-d_{13}\right) y_{6}-\left(b_{2}-c-d_{23}\right) y_{3}}{\left(b_{2}+c+d_{23}\right) y_{6}-\left(b_{2}+d_{12}+d_{13}\right) y_{3}} y_{2}, y_{2}
\end{array}\right), \\
\pi:\left(y_{1}, y_{2}, y_{3}, y_{4}, y_{5}, y_{6}\right) \mapsto\left(y_{3}, y_{5}, y_{6}, y_{2}, y_{4}, y_{1}\right) .
\end{gathered}
$$

Remark 2.1. We follow the convention that the parameters and $y$-variables not explicitly included in the actions listed in Equations (2.9) and (2.10) are the ones that remain unchanged under the action of the corresponding transformation. That is, the transformation acts as an identity on those parameters or variables.

For later convenience, we here define the translations in $\widetilde{W}\left(A_{2}^{(1)}\right)$ by

$$
T_{1}=w_{1} w_{2} \pi^{2}, \quad T_{2}=w_{2} w_{0} \pi^{2}, \quad T_{3}=w_{0} w_{1} \pi^{2},
$$

whose actions on the parameters $b_{0}, b_{1}, b_{2}, c, d_{12}, d_{23}, d_{13}$ are given by

$$
\begin{aligned}
& T_{1}:\left(b_{0}, b_{1}, d_{12}, d_{13}\right) \mapsto\left(b_{0}-1, b_{1}+1,-d_{12},-d_{13}\right), \\
& T_{2}:\left(b_{1}, b_{2}, d_{12}, d_{23}\right) \mapsto\left(b_{1}-1, b_{2}+1,-d_{12},-d_{23}\right), \\
& T_{3}:\left(b_{2}, b_{0}, d_{23}, d_{13}\right) \mapsto\left(b_{2}-1, b_{0}+1,-d_{23},-d_{13}\right) .
\end{aligned}
$$

Note that $T_{1} T_{2} T_{3}=1$ and $T_{i} T_{j}=T_{j} T_{i}$, where $i, j=1,2,3$. 
2.2. Derivation of the partial difference equations from $\widetilde{W}\left(A_{2}^{(1)}\right)$. In this subsection, we derive the $\mathrm{P} \Delta \mathrm{Es}(2.16)$ from the birational action of $\widetilde{W}\left(A_{2}^{(1)}\right)$.

Let

$$
u_{l_{1}, l_{2}, l_{3}}=T_{1}^{l_{1}} T_{2}^{l_{2}} T_{3}^{l_{3}}\left(y_{2}\right) .
$$

Note that

$u_{0,1,1}=y_{1}, \quad u_{0,0,0}=y_{2}, \quad u_{1,0,0}=y_{3}, \quad u_{0,1,0}=y_{4}, \quad u_{1,1,0}=y_{5}, \quad u_{1,2,0}=y_{6}$.

We assign the variable $u_{l_{1}, l_{2}, l_{3}}$ on the vertices $\left(l_{1}, l_{2}, l_{3}\right)$ of the triangle lattice

$$
\mathbb{Z}^{3} /(1,1,1):=\left\{\left(l_{1}, l_{2}, l_{3}\right) \in \mathbb{Z}^{3} \mid l_{1}+l_{2}+l_{3}=0\right\} .
$$

Then, we obtain Lemma 2.2 .

Lemma 2.2. On the triangle lattice there are three fundamental relations (essentially two):

$$
\frac{u_{\bar{i}}}{u_{\underline{i}}}=\frac{\left(b_{l_{i}, l_{j}}^{(i)}-c+(-1)^{l_{i}+l_{j}} d_{i j}\right) u_{\bar{j}}-\left(b_{l_{i}, l_{j}}^{(i)}-(-1)^{l_{j}+l_{k}} d_{j k}+(-1)^{l_{i}+l_{k}} d_{i k}\right) u_{\underline{j}}}{\left(b_{l_{i}, l_{j}}^{(i)}+(-1)^{l_{j}+l_{k}} d_{j k}-(-1)^{l_{i}+l_{k}} d_{i k}\right) u_{\bar{j}}-\left(b_{l_{i}, l_{j}}^{(i)}+c-(-1)^{l_{i}+l_{j}} d_{i j}\right) u_{\underline{j}}},
$$

where $(i, j, k)=(1,2,3),(2,3,1),(3,1,2)$ and

$$
b_{l_{1}, l_{2}}^{(1)}=b_{1}+l_{1}-l_{2}, \quad b_{l_{2}, l_{3}}^{(2)}=b_{2}+l_{2}-l_{3}-1, \quad b_{l_{1}, l_{3}}^{(0)}=b_{0}+l_{3}-l_{1} .
$$

Here, $u=u_{l_{1}, l_{2}, l_{3}}$ and the subscript $\bar{i}$ (or, $\underline{i}$ ) for a function $u=u_{l_{1}, l_{2}, l_{3}}$ means +1 shift (or, -1 shift) in the $l_{i}$-direction.

Proof. Equation (2.16) with $(i, j, k)=(1,2,3),(2,3,1),(3,1,2)$ are respectively obtained from the following actions:

$$
\begin{aligned}
& \frac{T_{1}\left(y_{5}\right)}{y_{4}}=\frac{\left(b_{1}-c+d_{12}\right) y_{6}-\left(b_{1}+d_{23}-d_{13}\right) y_{3}}{\left(b_{1}-d_{23}+d_{13}\right) y_{6}-\left(b_{1}+c-d_{12}\right) y_{3}}, \\
& \frac{T_{2}\left(y_{4}\right)}{y_{2}}=\frac{\left(b_{2}-c-d_{23}\right) y_{1}-\left(b_{2}-d_{13}-d_{12}\right) y_{6}}{\left(b_{2}+d_{13}+d_{12}\right) y_{1}-\left(b_{2}+c+d_{23}\right) y_{6}}, \\
& \frac{T_{3}\left(y_{2}\right)}{y_{5}}=\frac{\left(b_{0}-c+d_{13}\right) y_{3}-\left(b_{0}-d_{12}+d_{23}\right) y_{1}}{\left(b_{0}+d_{12}-d_{23}\right) y_{3}-\left(b_{0}+c-d_{13}\right) y_{1}} .
\end{aligned}
$$

Moreover, we can easily verify that using Equations (2.16) we can express any $u_{l_{1}, l_{2}, l_{3}}$ on the lattice by the six initial variables $y_{i}, i=1, \ldots, 6$, and one of the equations (2.16) can be obtained from the other two equations. Therefore, we have completed the proof. 
Remark 2.3. Because of the following relations:

(2.19a)

$$
w_{0}\left(u_{l_{1}, l_{2}, l_{3}}\right)=u_{l_{3}, l_{2}, l_{1}}, \quad w_{1}\left(u_{l_{1}, l_{2}, l_{3}}\right)=u_{l_{2}, l_{1}, l_{3}}, \quad w_{2}\left(u_{l_{1}, l_{2}, l_{3}}\right)=u_{l_{1}+1, l_{3}+2, l_{2}},
$$

$$
\pi\left(u_{l_{1}, l_{2}, l_{3}}\right)=u_{l_{3}+1, l_{1}+1, l_{2}},
$$

which follow from

$$
\begin{aligned}
& w_{0} T_{\{1,2,3\}}=T_{\{3,2,1\}} w_{0}, \quad w_{1} T_{\{1,2,3\}}=T_{\{2,1,3\}} w_{1}, \quad w_{2} T_{\{1,2,3\}}=T_{\{1,3,2\}} w_{2}, \\
& \pi T_{\{1,2,3\}}=T_{\{2,3,1\}} \pi, \quad w_{0}\left(u_{0,0,0}\right)=u_{0,0,0}, \quad w_{1}\left(u_{0,0,0}\right)=u_{0,0,0}, \\
& w_{2}\left(u_{0,0,0}\right)=u_{1,2,0}, \quad \pi\left(u_{0,0,0}\right)=u_{1,1,0},
\end{aligned}
$$

the transformation group $\widetilde{W}\left(A_{2}^{(1)}\right)$ can be also regarded as a symmetry of the triangle lattice (see Figure 2.1).

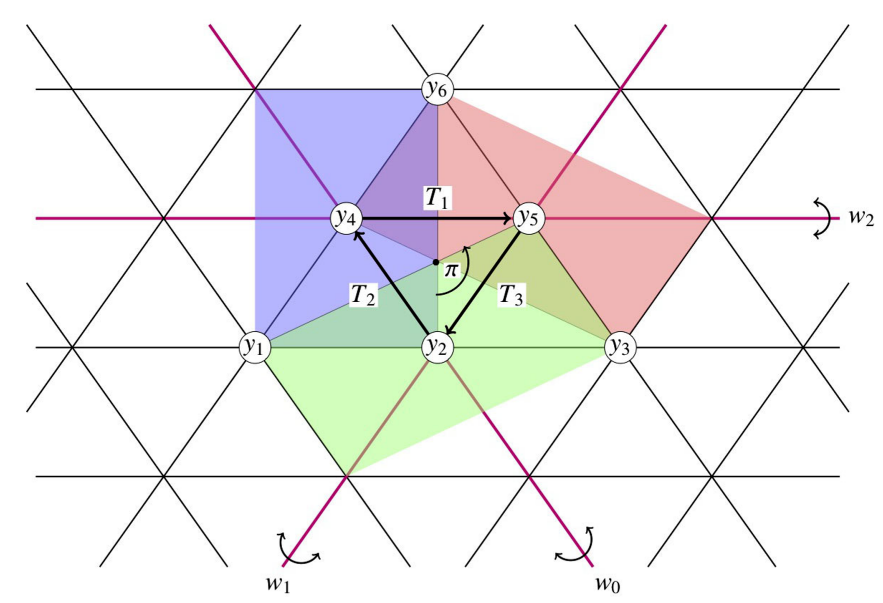

FIGURE 2.1. Triangle lattice. On the vertices the variables $u_{l_{1}, l_{2}, l_{3}}$ are assigned, and on the quadrilaterals there exist quad-equations (2.16), e.g. Equations (2.18a), (2.18b) and (2.18c) are colored in red, blue and green, respectively.

2.3. Derivation of the $A_{2}^{(1) *}$-type discrete Painlevé equations from $\widetilde{W}\left(A_{2}^{(1)}\right)$. In this subsection, we derive the $A_{2}^{(1) *}$-type discrete Painlevé equations (1.4) from the birational action of $\widetilde{W}\left(A_{2}^{(1)}\right)$.

Let

$$
\begin{aligned}
& f=\frac{\left(c-d_{12}+d_{23}-d_{13}\right) y_{1}}{2\left(y_{6}-y_{1}\right)}+\frac{b_{2}+c-d_{12}+d_{23}-d_{13}}{4}, \\
& g=\frac{\left(c-d_{12}+d_{23}-d_{13}\right) y_{3}}{2\left(y_{3}-y_{6}\right)}-\frac{b_{2}+c-d_{12}+d_{23}-d_{13}}{4} .
\end{aligned}
$$


Then, the action of $\widetilde{W}\left(A_{2}^{(1)}\right)$ on the variables $f$ and $g$ is given by

$$
\begin{aligned}
& w_{0}(f)=f-\frac{b_{0}}{4}, \quad w_{1}(g)=g+\frac{b_{1}}{4}, \quad \pi(g)=f-\frac{b_{2}+b_{0}}{4}, \\
& \frac{4\left(c-d_{12}+d_{23}-d_{13}\right)}{4 f-2 b_{0}-b_{2}-c-d_{12}+d_{23}+d_{13}}\left(w_{0}(g)+\frac{b_{2}+b_{0}+c+d_{12}-d_{23}-d_{13}}{4}\right) \\
& =\frac{\left(b_{0}-d_{12}+d_{23}\right)\left(4 g+b_{2}-c+d_{12}-d_{23}+d_{13}\right)}{4 f-b_{2}+c-d_{12}+d_{23}-d_{13}} \\
& -\frac{\left(b_{0}-c+d_{13}\right)\left(4 g+b_{2}+c-d_{12}+d_{23}-d_{13}\right)}{4 f-b_{2}-c+d_{12}-d_{23}+d_{13}}, \\
& \frac{4\left(c-d_{12}+d_{23}-d_{13}\right)}{4 g+2 b_{1}+b_{2}+c-d_{12}-d_{23}+d_{13}}\left(w_{1}(f)-\frac{b_{1}+b_{2}+c-d_{12}-d_{23}+d_{13}}{4}\right) \\
& =\frac{\left(b_{1}+d_{23}-d_{13}\right)\left(4 f-b_{2}+c-d_{12}+d_{23}-d_{13}\right)}{4 g+b_{2}-c+d_{12}-d_{23}+d_{13}} \\
& -\frac{\left(b_{1}-c+d_{12}\right)\left(4 f-b_{2}-c+d_{12}-d_{23}+d_{13}\right)}{4 g+b_{2}+c-d_{12}+d_{23}-d_{13}}, \\
& \pi(f)=-\frac{\left(4 f-b_{2}+c-d_{12}+d_{23}-d_{13}\right)\left(4 g+b_{2}+c-d_{12}+d_{23}-d_{13}\right)}{16(f+g)} \\
& +\frac{b_{0}+c-d_{12}+d_{23}-d_{13}}{4} .
\end{aligned}
$$

Using the transformation $T_{1}^{2}$ whose action on the parameter space $\left\{b_{0}, b_{1}, b_{2}, c, d_{12}\right.$, $\left.d_{23}, d_{13}\right\}$ is translational as $T_{1}^{2}:\left(b_{0}, b_{1}\right) \mapsto\left(b_{0}-2, b_{1}+2\right)$ shows, we obtain the discrete Painlevé equation (1.4a) with the following correspondence:

$$
\begin{aligned}
& X=f, \quad Y=g, \quad \bar{X}=T_{1}^{2}(f), \quad \underline{Y}=T_{1}^{-2}(g), \quad t=\frac{2 b_{1}+b_{2}-2}{4}, \\
& c_{1}=\frac{\left(b_{2}+c+d_{23}\right)^{2}}{16}, \quad c_{2}=\frac{\left(b_{2}-c-d_{23}\right)^{2}}{16}, \quad c_{3}=\frac{d_{12}+d_{13}}{4}, \\
& c_{4}=\frac{\left(c+d_{12}-d_{23}-d_{13}\right)^{2}}{16}, \quad c_{5}=\frac{\left(c-d_{12}-d_{23}+d_{13}\right)^{2}}{16} .
\end{aligned}
$$

We can also obtain the discrete Painlevé equations from another operation on the parameter space as follows [12]. The action of $T_{1}$ on the parameter space:

$$
T_{1}:\left(b_{0}, b_{1}, d_{12}, d_{13}\right) \mapsto\left(b_{0}-1, b_{1}+1,-d_{12},-d_{13}\right),
$$

is not translational, but when the parameters take the special values $d_{12}=d_{13}=0$, it becomes translational motion on the parameter sub-space $\left\{b_{0}, b_{1}, b_{2}, c, d_{23}\right\}: T_{1}$ : $\left(b_{0}, b_{1}\right) \mapsto\left(b_{0}-1, b_{1}+1\right)$. Under the specialization of the parameters, the action of $T_{1}$ gives the discrete Painlevé equation (1.4b) with the following correspondence:

$$
\begin{aligned}
& X=2 f, \quad \bar{X}=T_{1}(2 f), \quad \underline{X}=T_{1}^{-1}(2 f), \quad t=\frac{2 b_{1}+b_{2}-2}{2}, \\
& c_{1}=\frac{\left(b_{2}+c+d_{23}\right)^{2}}{4}, \quad c_{2}=\frac{\left(b_{2}-c-d_{23}\right)^{2}}{4}, \quad c_{3}=\frac{\left(c-d_{23}\right)^{2}}{4} .
\end{aligned}
$$

\section{Proof of Theorem 1.1}

In this section, we give the proof of Theorem 1.1 via the reduction from the system of $\mathrm{P} \Delta \mathrm{Es}(1.2)$ to the system of $\mathrm{P} \Delta \mathrm{Es}(2.16)$.

Lemma 3.1 holds. 
Lemma 3.1. By imposing the $(1,1,1)$-periodic condition: $u\left(\boldsymbol{l}+\boldsymbol{\epsilon}_{1}+\boldsymbol{\epsilon}_{2}+\boldsymbol{\epsilon}_{3}\right)=u(\boldsymbol{l})$ for $\boldsymbol{l} \in \Omega$, the system (1.2) can be reduced to the following system of PDEs:

$$
\frac{u_{\bar{i}}}{u_{\underline{i}}}=\frac{\left(\alpha_{i j}-c+(-1)^{l_{i}+l_{j}} \delta_{i}\right) u_{\bar{j}}-\left(\alpha_{i j}-(-1)^{l_{j}+l_{k}} \delta_{j}+(-1)^{l_{i}+l_{k}} \delta_{k}\right) u_{\underline{j}}}{\left(\alpha_{i j}+(-1)^{l_{j}+l_{k}} \delta_{j}-(-1)^{l_{i}+l_{k}} \delta_{k}\right) u_{\bar{j}}-\left(\alpha_{i j}+c-(-1)^{l_{i}+l_{j}} \delta_{i}\right) u_{\underline{j}}},
$$

where $(i, j, k)=(1,2,3),(2,3,1),(3,1,2), u=u(\boldsymbol{l})$ and $\boldsymbol{l}=\sum_{i=1}^{3} l_{i} \boldsymbol{\epsilon}_{i} \in \mathbb{Z}^{3} /\left(\boldsymbol{\epsilon}_{1}+\right.$ $\left.\epsilon_{2}+\epsilon_{3}\right)$.

Proof. Applying the (1,1,1)-periodic condition to the system (1.2), we obtain Equation (3.1) with $(i, j, k)=(1,2,3),(2,3,1)$ and $(3,1,2)$ from Equation (1.2) with $(i, j, k)=(1,2,3),(2,3,1)$ and $(3,1,2)$, respectively. Therefore, we have completed the proof.

\section{Remark 3.2.}

(i) The number of essential equations in the system (3.1) is two.

(ii) By the (1,1,1)-reduction, each cuboctahedron is reduced to a hexagram (see Figure 3.1), which causes the reduction from the face-centred cubic lattice $\Omega$ to the triangle lattice $\mathbb{Z}^{3} /\left(\boldsymbol{\epsilon}_{1}+\boldsymbol{\epsilon}_{2}+\boldsymbol{\epsilon}_{3}\right)$.
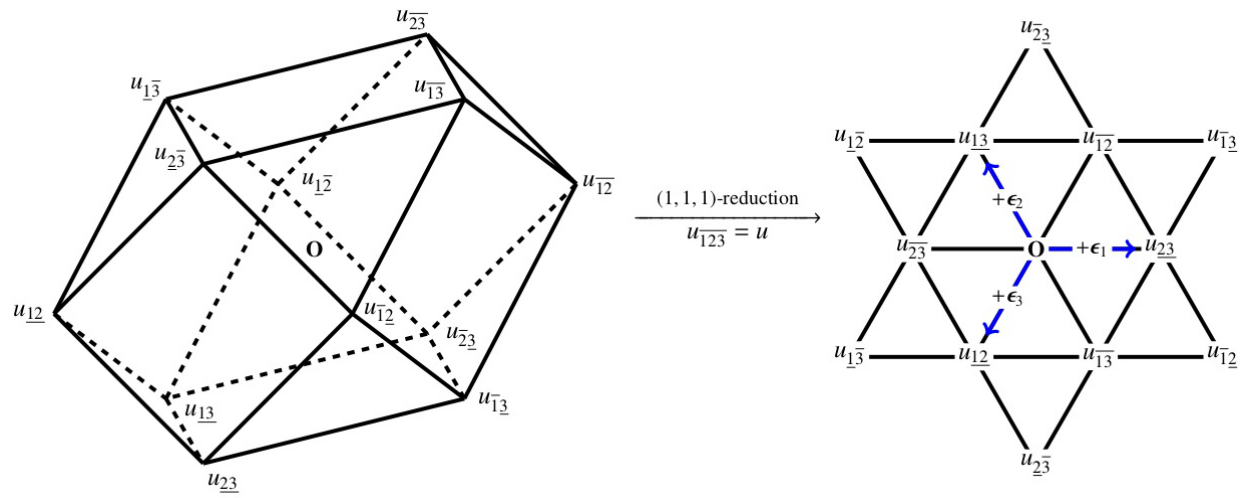

Figure 3.1. The $(1,1,1)$-reduction of the cuboctahedron

Lemma 3.3. The reduced system (3.1) is equivalent to equations in the system (2.16).

Proof. The statement follows from the following correspondences:

$$
\begin{aligned}
& b_{l_{1}, l_{2}}^{(1)}=\alpha_{12}, \quad b_{l_{2}, l_{3}}^{(2)}=\alpha_{23}, \quad b_{l_{1}, l_{3}}^{(0)}=\alpha_{31}, \quad d_{12}=\delta_{1}, \quad d_{23}=\delta_{2}, \quad d_{13}=\delta_{3}, \\
& u_{l_{1}, l_{2}, l_{3}}=u\left(l_{1} \epsilon_{1}+l_{2} \epsilon_{2}+l_{3} \epsilon_{3}\right) .
\end{aligned}
$$

Remark 3.4. Lemma 3.3 means that the reduced system (3.1) can be obtained from the theory of the $\tau$-function associated with $A_{2}^{(1) *}$-type discrete Painlevé equations. 
We are now ready to prove Theorem 1.1. The $(1,1,1)$-periodic reduction from the system (1.2) to the system (3.1) given in Lemma 3.1 the relation between the system (3.1) and the system (2.16) given in Lemma 3.3, and that between the system (2.16) and the $A_{2}^{(1) *}$-type discrete Painlevé equations (1.4) given in $\$ 2.2$ and 22.3 collectively give the proof of Theorem 1.1

\section{Concluding REMARKS}

In this paper, we considered a reduction of a system of $\mathrm{P} \Delta \mathrm{Es}$, which is unusual in the sense that it has the CACO property but not the widely studied CAC property. We showed how the system (1.2) can be reduced to the $A_{2}^{(1) *}$-type discrete Painlevé equations (1.4) using the affine Weyl group associated with the discrete Painlevé equations.

In a forthcoming paper (N. Joshi and N. Nakazono), we will show how another system of P $\Delta$ Es, which also has the CACO property, can be reduced to the $A_{2}^{(1)}$ type discrete Painlevé equations (see Table 1.1 for the distinction between $A_{2}^{(1)}$ and $\left.A_{2}^{(1) *}\right)$.

\section{Appendix A. Consistency around a Cuboctahedron property}

In this appendix, we recall the definition of consistency around a cuboctahedron. To define it, we also introduce an additional important property called consistency around an octahedron. We refer the reader to [10] for detailed information about these properties.

A.1. Consistency around an octahedron property. In this subsection, we give a definition of a consistency around an octahedron.

Let $u_{i}, i=1, \ldots, 6$, be variables and consider the octahedron shown in Figure A.1. The planes that pass through the vertices $\left\{u_{4}, u_{2}, u_{1}, u_{5}\right\},\left\{u_{2}, u_{6}, u_{5}, u_{3}\right\}$ and $\left\{u_{6}, u_{4}, u_{3}, u_{1}\right\}$ give 3 quadrilaterals that lie in the interior of the octahedron and we assign the quad-equations $Q_{i}, i=1,2,3$, to the quadrilaterals as the following:

$$
Q_{1}\left(u_{4}, u_{2}, u_{1}, u_{5}\right)=0, \quad Q_{2}\left(u_{2}, u_{6}, u_{5}, u_{3}\right)=0, \quad Q_{3}\left(u_{6}, u_{4}, u_{3}, u_{1}\right)=0 .
$$

The consistency around an octahedron property is defined by the following.

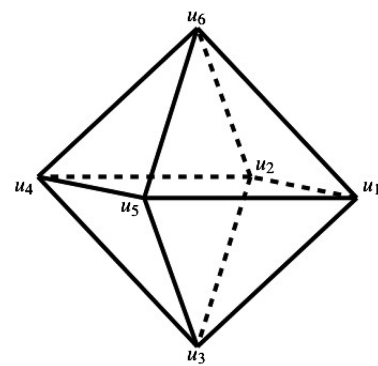

Figure A.1. An octahedron labelled with vertices $u_{i}, i=1, \ldots, 6$ 
Definition A.1 (CAO property [10]). The octahedron with quad-equations $\left\{Q_{1}\right.$, $\left.Q_{2}, Q_{3}\right\}$ is said to have a consistency around an octahedron (CAO) property if each quad-equation can be obtained from the other two equations. An octahedron is said to be a $C A O$ octahedron if it has the CAO property.

A.2. Consistency around a cuboctahedron property. In this subsection, we give a definition of consistency around a cuboctahedron.

We consider the cuboctahedron centered around the origin whose twelve vertices are given by $V=\left\{ \pm \boldsymbol{\epsilon}_{i} \pm \boldsymbol{\epsilon}_{j} \mid i, j \in \mathbb{Z}, 1 \leq i<j \leq 3\right\}$, where $\left\{\boldsymbol{\epsilon}_{1}, \boldsymbol{\epsilon}_{2}, \boldsymbol{\epsilon}_{3}\right\}$ form the standard basis of $\mathbb{R}^{3}$. We assign the variables $u(\boldsymbol{l})$ to the vertices $\boldsymbol{l} \in V$ and impose the following relations:

(A.2a) $Q_{1}\left(u_{5}, u_{1}, v_{5}, v_{4}\right)=0, \quad Q_{2}\left(v_{2}, v_{1}, u_{2}, u_{4}\right)=0, \quad Q_{3}\left(u_{3}, u_{5}, v_{3}, v_{2}\right)=0$,

(A.2b) $Q_{4}\left(v_{6}, v_{5}, u_{6}, u_{2}\right)=0, \quad Q_{5}\left(u_{1}, u_{3}, v_{1}, v_{6}\right)=0, \quad Q_{6}\left(v_{4}, v_{3}, u_{4}, u_{6}\right)=0$,

(A.2c) $Q_{7}\left(u_{4}, u_{2}, u_{1}, u_{5}\right)=0, \quad Q_{8}\left(u_{2}, u_{6}, u_{5}, u_{3}\right)=0, \quad Q_{9}\left(u_{6}, u_{4}, u_{3}, u_{1}\right)=0$,

where $Q_{i}, i=1, \ldots, 9$, are quad-equations and

$$
u_{1}=u\left(\boldsymbol{\epsilon}_{2}+\boldsymbol{\epsilon}_{3}\right), \quad u_{2}=u\left(-\boldsymbol{\epsilon}_{1}-\boldsymbol{\epsilon}_{3}\right), \quad u_{3}=u\left(\boldsymbol{\epsilon}_{1}+\boldsymbol{\epsilon}_{2}\right), \quad u_{4}=u\left(-\boldsymbol{\epsilon}_{2}-\boldsymbol{\epsilon}_{3}\right),
$$

$$
u_{5}=u\left(\boldsymbol{\epsilon}_{1}+\boldsymbol{\epsilon}_{3}\right), \quad u_{6}=u\left(-\boldsymbol{\epsilon}_{1}-\boldsymbol{\epsilon}_{2}\right), \quad v_{1}=u\left(\boldsymbol{\epsilon}_{2}-\boldsymbol{\epsilon}_{3}\right), \quad v_{2}=u\left(\boldsymbol{\epsilon}_{1}-\boldsymbol{\epsilon}_{3}\right),
$$

$$
v_{3}=u\left(\boldsymbol{\epsilon}_{1}-\boldsymbol{\epsilon}_{2}\right), \quad v_{4}=u\left(-\boldsymbol{\epsilon}_{2}+\boldsymbol{\epsilon}_{3}\right), \quad v_{5}=u\left(-\boldsymbol{\epsilon}_{1}+\boldsymbol{\epsilon}_{3}\right), \quad v_{6}=u\left(-\boldsymbol{\epsilon}_{1}+\boldsymbol{\epsilon}_{2}\right) .
$$

Note that quad-equations $Q_{i}, i=1, \ldots, 6$, are assigned to the faces of the cuboctahedron (see Figure A.2a). Moreover, $u_{i}, i=1, \ldots, 6$, collectively form the vertices of an octahedron and quad-equations $Q_{i}, i=7,8,9$, are assigned to the quadrilaterals that appear as sections passing through four vertices of the octahedron (see Figure A.2b).

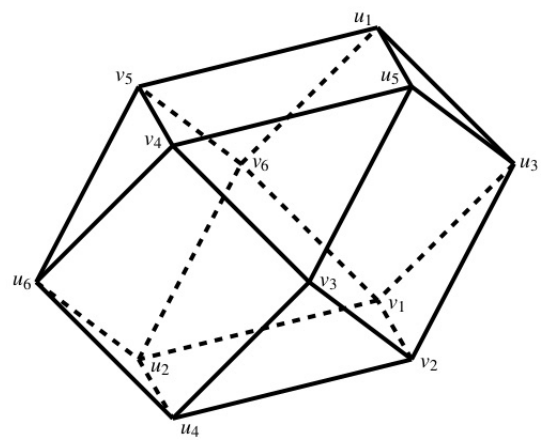

(a) A cuboctahedron labelled with vertices $u_{i}$ and $v_{j}, i, j=1, \ldots, 6$

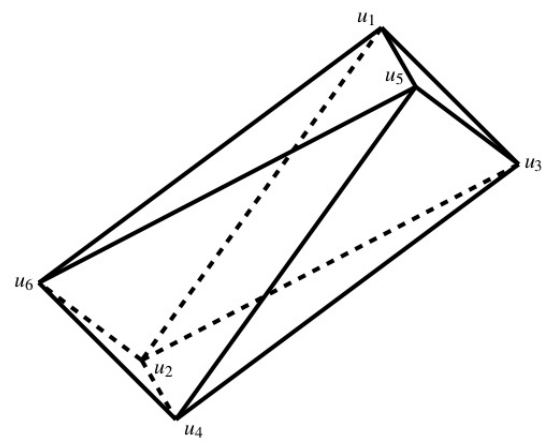

(a) An octahedron labelled with vertices $u_{i}, i=1, \ldots, 6$

Figure A.2. A cuboctahedron and an interior octahedron 
We are now in a position to give the following definitions.

Definition A.2 (CACO property [10]). The cuboctahedron with quad-equations $\left\{Q_{1}, \ldots, Q_{9}\right\}$ is said to have a consistency around a cuboctahedron (CACO) property if the following properties hold.

(i) The octahedron with quad-equations $\left\{Q_{7}, Q_{8}, Q_{9}\right\}$ has the CAO property.

(ii) Assume that $u_{1}, \ldots, u_{6}$ are given so as to satisfy $Q_{i}=0, i=7,8,9$, and, in addition, $v_{k}$ is given, for some $k \in\{1, \ldots, 6\}$. Then, quad-equations $Q_{i}$, $i=1 \ldots, 6$, determine the variables $v_{j}, j \in\{1, \ldots, 6\} \backslash\{k\}$, uniquely.

A cuboctahedron is said to be a $C A C O$ cuboctahedron if it has the CACO property.

Definition A.3 (Square property 10]). The CACO cuboctahedron with quadequations $\left\{Q_{1}, \ldots, Q_{9}\right\}$ is said to have a square property if there exist polynomials $K_{i}=K_{i}(x, y, z, w), i=1,2,3$, where $\operatorname{deg}_{x} K_{i}=\operatorname{deg}_{w} K_{i}=1$ and $1 \leq$ $\operatorname{deg}_{y} K_{i}, \operatorname{deg}_{z} K_{i}$, satisfying

$$
K_{1}\left(v_{1}, u_{1}, u_{4}, v_{4}\right)=0, \quad K_{2}\left(v_{2}, u_{2}, u_{5}, v_{5}\right)=0, \quad K_{3}\left(v_{3}, u_{3}, u_{6}, v_{6}\right)=0 .
$$

Then, each equation $K_{i}=0$ is called a square equation.

A.3. CACO property of $\mathbf{P} \Delta \mathbf{E s}$. We now explain how to associate quad-equations with $\mathrm{P} \Delta \mathrm{Es}$ in three-dimensional space by using the system of $\mathrm{P} \Delta \mathrm{Es}(1.2)$ as an example. This requires us to consider overlapping cuboctahedra that lead to twodimensional tessellations consisting of quadrilaterals. For each given cuboctahedron, there are twelve overlapping cuboctahedra.

The twelve overlapping cuboctahedra around a given one provide six directions of tiling by quadrilaterals. For later convenience, we label directions by $\boldsymbol{\epsilon}_{i} \pm \boldsymbol{\epsilon}_{j}$, $1 \leq i<j \leq 3$. Vertices labelled in this way form the set $\Omega$ given by (1.1). Such vertices are interpreted as being iterated on each successive cuboctahedron. We here consider the system of $\mathrm{P} \Delta \mathrm{Es}$ (1.2). For simplicity, we abbreviate each respective equation in Equations (1.2) as

$$
\begin{aligned}
& P_{1}\left(u_{\overline{13}}, u_{\overline{23}}, u_{\underline{1} \overline{3}}, u_{\underline{\underline{3}}}\right)=0, P_{2}\left(u_{\overline{12}}, u_{\overline{13}}, u_{\overline{1} \underline{2}}, u_{\overline{1} \underline{3}}\right)=0, P_{3}\left(u_{\overline{23}}, u_{\overline{12}}, u_{\overline{2} \underline{3}}, u_{\underline{1} \overline{2}}\right)=0, \\
& P_{4}\left(u_{\underline{23}}, u_{\underline{13}}, u_{\overline{23}}, u_{\overline{13}}\right)=0, P_{5}\left(u_{\underline{13}}, u_{\underline{12}}, u_{\overline{13}}, u_{\overline{12}}\right)=0, P_{6}\left(u_{\underline{12}}, u_{\underline{23}}, u_{\overline{12}}, u_{\overline{23}}\right)=0 .
\end{aligned}
$$

Conversely, given $\boldsymbol{l} \in \Omega$, we obtain the cuboctahedron centered around $\boldsymbol{l}$. We refer to its quad-equations as before by $\left\{Q_{1}(\boldsymbol{l}), \ldots, Q_{9}(\boldsymbol{l})\right\}$. Moreover, the overlapped region gives an octahedron centred around $\boldsymbol{l}+\boldsymbol{\epsilon}_{3}$, and we label its quad-equations by $\left\{\hat{Q}_{1}(\boldsymbol{l}), \hat{Q}_{2}(\boldsymbol{l}), \hat{Q}_{3}(\boldsymbol{l})\right\}$. 
Each such quad-equation is identified with the 6 partial difference equations given in Equations (1.2) in the following way. For $Q_{1}, \ldots, Q_{9}$, we use

$$
\begin{array}{ll}
Q_{1}(\boldsymbol{l})=P_{1}\left(u_{\overline{13}}, u_{\overline{23}}, u_{\underline{1} \overline{3}}, u_{\underline{2} \overline{3}}\right)=0, & Q_{2}(\boldsymbol{l})=P_{1}\left(u_{\overline{1} \underline{3}}, u_{\overline{1} \underline{3}}, u_{\underline{13}}, u_{\underline{23}}\right)=0, \\
Q_{3}(\boldsymbol{l})=P_{2}\left(u_{\overline{12}}, u_{\overline{13}}, u_{\overline{1} \underline{2}}, u_{\overline{1} \underline{3}}\right)=0, & Q_{4}(\boldsymbol{l})=P_{2}\left(u_{\underline{1} \overline{2}}, u_{\underline{1} \overline{3}}, u_{\underline{12}}, u_{\underline{13}}\right)=0, \\
Q_{5}(\boldsymbol{l})=P_{3}\left(u_{\overline{23}}, u_{\overline{12}}, u_{\overline{2} \underline{3}}, u_{\underline{1} \overline{2}}\right)=0, & Q_{6}(\boldsymbol{l})=P_{3}\left(u_{\underline{2} \overline{3}}, u_{\overline{1} \underline{2}}, u_{\underline{23}}, u_{\underline{12}}\right)=0, \\
Q_{7}(\boldsymbol{l})=P_{4}\left(u_{\underline{23}}, u_{\underline{13}}, u_{\overline{23}}, u_{\overline{13}}\right)=0, & Q_{8}(\boldsymbol{l})=P_{5}\left(u_{\underline{13}}, u_{\underline{12}}, u_{\overline{13}}, u_{\overline{12}}\right)=0, \\
Q_{9}(\boldsymbol{l})=P_{6}\left(u_{\underline{12}}, u_{\underline{23}}, u_{\overline{12}}, u_{\overline{23}}\right)=0, &
\end{array}
$$

and for $\hat{Q}_{1}, \hat{Q}_{2}, \hat{Q}_{3}$, we use

$$
\begin{aligned}
& \hat{Q}_{1}(\boldsymbol{l})=P_{1}\left(u_{\overline{13}}, u_{\overline{23}}, u_{\underline{1} \overline{3}}, u_{\underline{2} \overline{3}}\right)=0, \quad \hat{Q}_{2}(\boldsymbol{l})=P_{2}\left(u_{\overline{23}}, u_{\overline{33}}, u_{\underline{2} \overline{3}}, u\right)=0, \\
& \hat{Q}_{3}(\boldsymbol{l})=P_{3}\left(u_{\overline{33}}, u_{\overline{13}}, u, u_{\underline{1} \overline{3}}\right)=0 .
\end{aligned}
$$

Then, Proposition A.4 holds.

Proposition A.4 ([10]). The system of PAEs (1.2) has the CACO and square properties, that is, the following statements hold.

(i) The cuboctahedra with quad-equations $\left\{Q_{i}(\boldsymbol{l})\right\}$ have the CACO and square properties.

(ii) The square equations are consistent with the $P \Delta E s$ (1.2).

(iii) The octahedra with quad-equations $\left\{\hat{Q}_{i}(\boldsymbol{l})\right\}$ have the CAO property.

Appendix B. Extended AFFine Weyl group of type $E_{6}^{(1)}$ AND $\tau$-VARIABles

In this appendix, we review the action of the extended affine Weyl group of type $E_{6}^{(1)}$ given in 23], which is the symmetry group of $A_{2}^{(1) *}$-type discrete Painlevé equations.

Let $a_{i}, i=0, \ldots, 6$, be parameters satisfying the condition (2.1) and $\tau_{j}^{(i)}, i=$ $1,2,3, j=0,1,2,3$, be variables. The actions of transformations $s_{i}, i=0, \ldots, 6$, and $\iota_{j}, j=1,2,3$, on the parameters are given by

$$
\begin{aligned}
& s_{0}:\left(a_{0}, a_{6}\right) \mapsto\left(-a_{0}, a_{6}+a_{0}\right), \quad s_{1}:\left(a_{1}, a_{2}\right) \mapsto\left(-a_{1}, a_{2}+a_{1}\right), \\
& s_{2}:\left(a_{1}, a_{2}, a_{3}\right) \mapsto\left(a_{1}+a_{2},-a_{2}, a_{3}+a_{2}\right), \\
& s_{3}:\left(a_{2}, a_{3}, a_{4}, a_{6}\right) \mapsto\left(a_{2}+a_{3},-a_{3}, a_{4}+a_{3}, a_{6}+a_{3}\right), \\
& s_{4}:\left(a_{3}, a_{4}, a_{5}\right) \mapsto\left(a_{3}+a_{4},-a_{4}, a_{5}+a_{4}\right), \quad s_{5}:\left(a_{4}, a_{5}\right) \mapsto\left(a_{4}+a_{5},-a_{5}\right), \\
& s_{6}:\left(a_{0}, a_{3}, a_{6}\right) \mapsto\left(a_{0}+a_{6}, a_{3}+a_{6},-a_{6}\right), \\
& \iota_{1} a_{\{0,5,4,6\}} \mapsto a_{\{5,0,4,6\}}, \quad \iota_{2} a_{\{0,1,2,6\}} \mapsto a_{\{1,0,6,2\}}, \quad \iota_{3} a_{\{1,5,2,4\}} \mapsto a_{\{5,1,4,2\}},
\end{aligned}
$$


while those on the $\tau$-variables $\tau_{j}^{(i)}, i=1,2,3, j=0,1,2,3$, are given by

$$
\begin{aligned}
& s_{0}:\left(\tau_{2}^{(3)}, \tau_{3}^{(3)}\right) \mapsto\left(\tau_{3}^{(3)}, \tau_{2}^{(3)}\right), \quad s_{1}:\left(\tau_{2}^{(1)}, \tau_{3}^{(1)}\right) \mapsto\left(\tau_{3}^{(1)}, \tau_{2}^{(1)}\right) \text {, } \\
& s_{2}:\left(\tau_{1}^{(1)}, \tau_{2}^{(1)}\right) \mapsto\left(\tau_{2}^{(1)}, \tau_{1}^{(1)}\right) \text {, } \\
& :\left(\tau_{0}^{(2)}, \tau_{0}^{(3)}\right) \mapsto\left(\frac{\left(a_{2}+a_{3}\right) \tau_{1}^{(1)} \tau_{0}^{(2)}-a_{2} \tau_{1}^{(2)} \tau_{0}^{(1)}}{a_{3} \tau_{2}^{(1)}}, \frac{\left(a_{2}+a_{3}\right) \tau_{1}^{(1)} \tau_{0}^{(3)}-a_{2} \tau_{1}^{(3)} \tau_{0}^{(1)}}{a_{3} \tau_{2}^{(1)}}\right) \text {, } \\
& s_{3}:\left(\tau_{1}^{(1)}, \tau_{1}^{(2)}, \tau_{1}^{(3)}, \tau_{0}^{(1)}, \tau_{0}^{(2)}, \tau_{0}^{(3)}\right) \mapsto\left(\tau_{0}^{(1)}, \tau_{0}^{(2)}, \tau_{0}^{(3)}, \tau_{1}^{(1)}, \tau_{1}^{(2)}, \tau_{1}^{(3)}\right) \text {, } \\
& s_{4}:\left(\tau_{1}^{(2)}, \tau_{2}^{(2)}\right) \mapsto\left(\tau_{2}^{(2)}, \tau_{1}^{(2)}\right) \text {, } \\
& :\left(\tau_{0}^{(1)}, \tau_{0}^{(3)}\right) \mapsto\left(\frac{\left(a_{3}+a_{4}\right) \tau_{1}^{(2)} \tau_{0}^{(1)}-a_{4} \tau_{1}^{(1)} \tau_{0}^{(2)}}{a_{3} \tau_{2}^{(2)}}, \frac{\left(a_{3}+a_{4}\right) \tau_{1}^{(2)} \tau_{0}^{(3)}-a_{4} \tau_{1}^{(3)} \tau_{0}^{(2)}}{a_{3} \tau_{2}^{(2)}}\right) \text {, } \\
& s_{5}:\left(\tau_{2}^{(2)}, \tau_{3}^{(2)}\right) \mapsto\left(\tau_{3}^{(2)}, \tau_{2}^{(2)}\right) \text {, } \\
& s_{6}:\left(\tau_{1}^{(3)}, \tau_{2}^{(3)}\right) \mapsto\left(\tau_{2}^{(3)}, \tau_{1}^{(3)}\right) \text {, } \\
& :\left(\tau_{0}^{(1)}, \tau_{0}^{(2)}\right) \mapsto\left(\frac{\left(a_{3}+a_{6}\right) \tau_{1}^{(3)} \tau_{0}^{(1)}-a_{6} \tau_{1}^{(1)} \tau_{0}^{(3)}}{a_{3} \tau_{2}^{(3)}}, \frac{\left(a_{3}+a_{6}\right) \tau_{1}^{(3)} \tau_{0}^{(2)}-a_{6} \tau_{1}^{(2)} \tau_{0}^{(3)}}{a_{3} \tau_{2}^{(3)}}\right), \\
& \iota_{1}:\left(\tau_{j}^{(2)}, \tau_{j}^{(3)}\right) \mapsto\left(\tau_{j}^{(3)}, \tau_{j}^{(2)}\right), \quad \iota_{2}:\left(\tau_{j}^{(1)}, \tau_{j}^{(3)}\right) \mapsto\left(\tau_{j}^{(3)}, \tau_{j}^{(1)}\right), \\
& \iota_{3}:\left(\tau_{j}^{(1)}, \tau_{j}^{(2)}\right) \mapsto\left(\tau_{j}^{(2)}, \tau_{j}^{(1)}\right), \quad j=0,1,2,3 .
\end{aligned}
$$

Remark B.1.

(i) Each transformation here defined is an isomorphism from the field of rational functions $K\left(\left\{\tau_{j}^{(i)}\right\}\right)$, where $K=\mathbb{C}\left(\left\{a_{i}\right\}\right)$, to itself.

(ii) We follow the convention of Remark 2.1 for the above equations. That is, each transformation acts as an identity on parameters or variables not appearing in its definition.

The transformations collectively form the extended affine Weyl group of type $E_{6}^{(1)}$, denoted by (2.2). Indeed, the following fundamental relations hold:

$$
\left(s_{i} s_{j}\right)^{A_{i j}}=1, \quad \iota_{1}{ }^{2}=\iota_{2}{ }^{2}=\iota_{3}{ }^{2}=1, \quad \iota_{1} \iota_{2}=\iota_{2} \iota_{3}=\iota_{3} \iota_{1}, \quad \iota_{2} \iota_{1}=\iota_{3} \iota_{2}=\iota_{1} \iota_{3},
$$

$$
\iota_{1} s_{\{0,5,4,6\}}=s_{\{5,0,4,6\}} \iota_{1}, \quad \iota_{2} s_{\{0,1,2,6\}}=s_{\{1,0,6,2\}} \iota_{2}, \quad \iota_{3} s_{\{1,5,2,4\}}=s_{\{5,1,4,2\}} \iota_{3},
$$

where $i, j \in\{0,1, \ldots, 6\}$ and

$$
\left(A_{i j}\right)_{i, j=0}^{6}=\left(\begin{array}{ccccccc}
2 & 0 & 0 & 0 & 0 & 0 & 3 \\
0 & 2 & 3 & 0 & 0 & 0 & 0 \\
0 & 3 & 2 & 3 & 0 & 0 & 0 \\
0 & 0 & 3 & 2 & 3 & 0 & 3 \\
0 & 0 & 0 & 3 & 2 & 3 & 0 \\
0 & 0 & 0 & 0 & 3 & 2 & 0 \\
3 & 0 & 0 & 3 & 0 & 0 & 2
\end{array}\right) .
$$

Remark B.2. The correspondence between the notations in this paper and those in [23] is given by $\tau_{j}^{(i)} \rightarrow \tau_{j}^{i}$ and $\tau_{0}^{(i)} \rightarrow s_{3}\left(\tau_{1}^{i}\right)$, where $i, j=1,2,3$. 


\section{ACKNOWLEDGMENTS}

The second author would like to thank Profs M. Noumi, Y. Ohta and Y. Yamada for inspiring and fruitful discussions.

\section{REFERENCES}

[1] V. E. Adler, A. I. Bobenko, and Yu. B. Suris, Classification of integrable equations on quadgraphs. The consistency approach, Comm. Math. Phys. 233 (2003), no. 3, 513-543, DOI 10.1007/s00220-002-0762-8. MR 1962121

[2] V. È. Adler, A. I. Bobenko, and Yu. B. Suris, Discrete nonlinear hyperbolic equations: classification of integrable cases (Russian, with Russian summary), Funktsional. Anal. i Prilozhen. 43 (2009), no. 1, 3-21, DOI 10.1007/s10688-009-0002-5; English transl., Funct. Anal. Appl. 43 (2009), no. 1, 3-17. MR2503862

[3] Raphael Boll, Classification of $3 D$ consistent quad-equations, J. Nonlinear Math. Phys. 18 (2011), no. 3, 337-365, DOI 10.1142/S1402925111001647. MR2846098

[4] Raphael Boll, Corrigendum: classification of 3D consistent quad-equations [MR2846098], J. Nonlinear Math. Phys. 19 (2012), no. 4, 1292001, 3, DOI 10.1142/S1402925112920015. MR.3010833

[5] R. Fuchs, Sur quelques équations différentielles linéaires du second ordre, C. R.Acad. des Sci. Paris 141 (1905), no. 1, 555-558.

[6] B. Gambier, Sur les équations différentielles du second ordre et du premier degré dont l'intégrale générale est a points critiques fixes (French), Acta Math. 33 (1910), no. 1, 155, DOI 10.1007/BF02393211. MR1555055

[7] B. Grammaticos and A. Ramani, Discrete Painlevé equations: a review, Discrete integrable systems, Lecture Notes in Phys., vol. 644, Springer, Berlin, 2004, pp. 245-321, DOI 10.1007/978-3-540-40357-9_7. MR2087743

[8] J. Hietarinta, N. Joshi, and F. W. Nijhoff, Discrete systems and integrability, Cambridge Texts in Applied Mathematics, Cambridge University Press, Cambridge, 2016, DOI 10.1017/CBO9781107337411. MR3587455

[9] Ryogo Hirota, Nonlinear partial difference equations. I. A difference analogue of the Korteweg-de Vries equation, J. Phys. Soc. Japan 43 (1977), no. 4, 1424-1433, DOI 10.1143/JPSJ.43.1424. MR460934

[10] N. Joshi and N. Nakazono, Classification of quad-equations on a cuboctahedron, arXiv:1906.06650 2019.

[11] K. Kajiwara, Hypergeometric solutions to the additive discrete Painleve equations with affine Weyl group symmetry of type E, Reports of RIAM Symposium (No. 19ME-S2), 2008 (in Japanese).

[12] Kenji Kajiwara, Nobutaka Nakazono, and Teruhisa Tsuda, Projective reduction of the discrete Painlevé system of type $\left(A_{2}+A_{1}\right)^{(1)}$, Int. Math. Res. Not. IMRN 4 (2011), 930-966, DOI 10.1093/imrn/rnq089. MR2773334

[13] Kenji Kajiwara, Masatoshi Noumi, and Yasuhiko Yamada, Geometric aspects of Painlevé equations, J. Phys. A 50 (2017), no. 7, 073001, 164, DOI 10.1088/1751-8121/50/7/073001. MR3609039

[14] Frank Nijhoff and Hans Capel, The discrete Korteweg-de Vries equation, Acta Appl. Math. 39 (1995), no. 1-3, 133-158, DOI 10.1007/BF00994631. KdV '95 (Amsterdam, 1995). MR.1329559

[15] F. W. Nijhoff, H. W. Capel, G. L. Wiersma, and G. R. W. Quispel, Bäcklund transformations and three-dimensional lattice equations, Phys. Lett. A 105 (1984), no. 6, 267-272, DOI 10.1016/0375-9601(84)90994-0. MR763123

[16] F. W. Nijhoff, G. R. W. Quispel, and H. W. Capel, Direct linearization of nonlinear difference-difference equations, Phys. Lett. A 97 (1983), no. 4, 125-128, DOI 10.1016/03759601(83)90192-5. MR719638

[17] F. W. Nijhoff and A. J. Walker, The discrete and continuous Painlevé VI hierarchy and the Garnier systems, Glasg. Math. J. 43A (2001), 109-123, DOI 10.1017/S0017089501000106. Integrable systems: linear and nonlinear dynamics (Islay, 1999). MR.1869690 
[18] J. J. C. Nimmo and W. K. Schief, An integrable discretization of a $(2+1)$-dimensional sine-Gordon equation, Stud. Appl. Math. 100 (1998), no. 3, 295-309, DOI 10.1111/14679590.00079. MR1609280

[19] P. Painlevé, Sur les équations différentielles du second ordre et d'ordre supérieur dont l'intégrale générale est uniforme (French), Acta Math. 25 (1902), no. 1, 1-85, DOI 10.1007/BF02419020. MR1554937

[20] G. R. W. Quispel, F. W. Nijhoff, H. W. Capel, and J. van der Linden, Linear integral equations and nonlinear difference-difference equations, Phys. A 125 (1984), no. 2-3, 344380, DOI 10.1016/0378-4371(84)90059-1. MR761644

[21] A. Ramani, B. Grammaticos, and J. Hietarinta, Discrete versions of the Painlevé equations, Phys. Rev. Lett. 67 (1991), no. 14, 1829-1832, DOI 10.1103/PhysRevLett.67.1829. MR.1125951

[22] Hidetaka Sakai, Rational surfaces associated with affine root systems and geometry of the Painlevé equations, Comm. Math. Phys. 220 (2001), no. 1, 165-229, DOI $10.1007 / \mathrm{s} 002200100446 . \mathrm{MR} 1882403$

[23] Teruhisa Tsuda, A geometric approach to tau-functions of difference Painlevé equations, Lett. Math. Phys. 85 (2008), no. 1, 65-78, DOI 10.1007/s11005-008-0251-x. MR2425662

School of Mathematics and Statistics F07, The University of Sydney, New South Wales 2006, Australia

Email address: nalini.joshi@sydney.edu.au

Institute of Engineering, Tokyo University of Agriculture and Technology, 2-24-16 NAKAChO KoganeI, TOKYO 184-8588, JAPAN

Email address: nakazono@go.tuat.ac.jp 